\title{
A PYTHAGOREAN APPROACH IN BANACH SPACES
}

JI GAO

Received 30 December 2003; Accepted 4 May 2004

Let $X$ be a Banach space and let $S(X)=\{x \in X,\|x\|=1\}$ be the unit sphere of $X$. Parameters $E(X)=\sup \{\alpha(x), x \in S(X)\}, e(X)=\inf \{\alpha(x), x \in S(X)\}, F(X)=\sup \{\beta(x), x \in$ $S(X)\}$, and $f(X)=\inf \{\beta(x), x \in S(X)\}$, where $\alpha(x)=\sup \left\{\|x+y\|^{2}+\|x-y\|^{2}, y \in\right.$ $S(X)\}$, and $\beta(x)=\inf \left\{\|x+y\|^{2}+\|x-y\|^{2}, y \in S(X)\right\}$ are introduced and studied. The values of these parameters in the $l_{p}$ spaces and function spaces $L_{p}[0,1]$ are estimated. Among the other results, we proved that a Banach space $X$ with $E(X)<8$, or $f(X)>2$ is uniform nonsquare; and a Banach space $X$ with $E(X)<5$, or $f(X)>32 / 9$ has uniform normal structure.

Copyright (c) 2006 Hindawi Publishing Corporation. All rights reserved.

\section{Preliminaries}

Let $X$ be a normed linear space, and let $S(X)=\{x \in X:\|x\|=1\}$ and $B(X)=\{x \in X$ : $\|x\| \leq 1\}$ be the unit sphere and unit ball of $X$ respectively.

The following two concepts, the modulus of convexity: $\delta(\epsilon)=\inf \{1-\|x+y\| / 2$ : $\left.\|x-y\| \leq \epsilon, x, y \in S\left(X_{2}\right)\right\}$ where $0 \leq \epsilon \leq 2$, and the modulus of smoothness: $\rho_{X}(\epsilon)=$ $\sup \{(\|x+y\|+\|x-y\|-2) / 2, x \in S(X),\|y\|=\epsilon\}$ where $\epsilon \geq 0$ have strong effect for studying and describing the shape of unit spheres and unit balls of Banach spaces.

Many other parameters were introduced and used to study the geometry of unit spheres and unit balls, more properties of Banach spaces were obtained and some results were improved, see $[6-8,11,12,14]$. However most of the above-defined parameters are linear in the norm.

The Pythagorean theorem describes the shape of unit sphere of Euclidean spaces $H$ by considering the inscribed triangle with two antipodal points $x$ and $-x$ on $S(H)$, and characterizes the Euclidean spaces by the equation $\|x+y\|^{2}+\|x-y\|^{2}=4$, for all $y \in S(H)$. In this paper we use this idea to introduce some quadratic parameters, then compute the values of these new parameters for some classical Banach spaces, and demonstrate the relationships between these parameters and uniformly nonsquare spaces, and the spaces with uniform normal structure. 
Let $X$ be a Banach space. A mapping $T: X \rightarrow X$ is called a nonexpansive mapping if $\|T x-T y\| \leq\|x-y\|$ for any $x, y \in X$. Kirk [10] proved that if a weakly compact convex subset $K$ of $X$ has normal structure then any nonexpansive mapping on $K$ has a fixed point.

Definition 1.1 [1]. A bounded convex subset $K$ of a Banach space $X$ is said to have normal structure if every convex subset $H$ of $K$ that contains more than one point contains a point $x_{0} \in H$ such that $\sup \left\{\left\|x_{0}-y\right\|, y \in H\right\}<d(H)$, where $d(H)=\sup \{\|x-y\|, x, y \in$ $H$ \} denotes the diameter of $H$. A Banach space $X$ is said to have normal structure if every bounded, convex subset of $X$ has normal structure. A Banach space $X$ is said to have weak normal structure if each weakly compact convex set $K$ in $X$ that contains more than one point has normal structure. $X$ is said to have uniform normal structure if there exists $0<c<1$ such that for any subset $K$ as above, there exists $x_{0} \in K$ such that $\sup \left\{\| x_{0}-\right.$ $y \|, y \in K\}<c \cdot(d(K))$.

For a reflexive Banach space $X$, the normal structure and weak normal structure coincide.

Definition 1.2 [5]. Let $X$ be a Banach space, a hexagon $H$ in $X$ is called a normal hexagon if the length of each side is 1 and each pair of two opposite sides are parallel.

Lemma 1.3 [5]. Let $X$ be a Banach space without weak normal structure, then for any $0<\epsilon<1$, there exist infinitely many inscribed normal hexagons with vertices $x_{1}, x_{2}, x_{3}$ in $S(X)$ satisfying

(i) $x_{2}-x_{3}=x_{1}$;

(ii) $\left\|\left(x_{1}+x_{2}\right) / 2\right\|>1-\epsilon$;

(iii) $\left\|\left(x_{3}+\left(-x_{1}\right)\right) / 2\right\|>1-\epsilon$.

A continuous mapping $x(t)$ from a closed interval $[a, b]$ to a Banach space $X$ is called a curve in $X: C=x(t), a \leq t \leq b$. A curve is called simple if it does not have multiple points. A curve is called closed if $x(a)=x(b)$. A closed curve is called symmetric about the origin if $x \in C$, then also $-x \in C$.

The concept of the length of a curve in Banach spaces resembles the same concept in Euclidean spaces.

For a normed linear space $X$, we use $X_{2}$ to denote a two-dimensional subspace of $X$, then $S\left(X_{2}\right)$ is a simple closed curve which is symmetric about the origin and unique up to orientation.

Theorem $1.4[2,12]$. Let $X_{2}$ be a two-dimensional Banach space, and $K_{1}, K_{2}$ be closed convex subsets of $X_{2}$, and have nonvoid interiors. If $K_{1} \subseteq K_{2}$, then $l\left(\partial\left(K_{1}\right)\right) \leq l\left(\partial\left(K_{2}\right)\right)$, where $l\left(\partial\left(K_{i}\right)\right)$ denote the lengths of the circumferences of $K_{i}, i=1,2$.

For $x \in S\left(X_{2}\right)$, let $\kappa$ be one of the arcs of $S\left(X_{2}\right)$ from $x$ to $-x$ counterclockwise, and let $g:[0, L] \rightarrow \kappa$ be the standard representation in terms of arc length, where $L$ is the length of $\kappa$.

Lemma 1.5 [7]. The functions $\Phi, \Psi:[0, L] \rightarrow[0,2]$ defined by $\Phi(s)=\|g(s)-x\|, \Psi(s)=$ $\|g(s)+x\|$ are continuously increasing, and decreasing respectively. 


\section{Parameters $E(X), e(X), F(X)$, and $f(X)$}

Definition 2.1. For $x \in S(X)$, let

(i) $\alpha(x)=\sup \left\{\|x+y\|^{2}+\|x-y\|^{2}, y \in S(X)\right\}$ and

(ii) $\beta(x)=\inf \left\{\|x+y\|^{2}+\|x-y\|^{2}, y \in S(X)\right\}$.

We define

$$
\begin{array}{ll}
E(X)=\sup \{\alpha(x), x \in S(X)\}, & e(X)=\inf \{\alpha(x), x \in S(X)\}, \\
F(X)=\sup \{\beta(x), x \in S(X)\}, & f(X)=\inf \{\beta(x), x \in S(X)\} .
\end{array}
$$

Lemma 2.2. If $a \geq 0, b \geq 0$, and $a+b \geq 2$, then $a^{2}+b^{2} \geq 2$.

Proof. $a^{2}+b^{2} \geq a^{2}+(2-a)^{2}=a^{2}+4-4 a+a^{2}=2\left(a^{2}-2 a+2\right) \geq 2\left[(a-1)^{2}+1\right] \geq 2$.

Proposition 2.3. For any Banach space $X, 2 \leq f(X) \leq e(X) \leq E(X) \leq 8$, and $2 \leq f(X) \leq$ $F(X) \leq E(X) \leq 8$.

Proof. Since $\|x \pm y\| \leq\|x\|+\|y\| \leq 2$, we have $E(X)=\sup \left\{\|x+y\|^{2}+\|x-y\|^{2}, x \in\right.$ $S(X), y \in S(X)\} \leq 4+4=8$; and since $\|x+y\|+\|x-y\| \geq\|2 x\|=2$, we have $f(X)=$ $\inf \left\{\|x+y\|^{2}+\|x-y\|^{2}, x \in S(X), y \in S(X)\right\} \geq 2$, by the about lemma.

We now list the values of these parameters for some special two-dimensional normed linear spaces. $X$.

For any $x \in X, y \in X$ we use $[x, y]$ to denote the line segment connecting $x$ and $y$ in

Lemma 2.4. Let $x, y \in S(X)$ with $\|x+y\|=\|x-y\|=a$, and $u$, $v$ be the normalization of $x+y, x-y$, respectively, then $\|u+v\|=\|u-v\|=2 / a$.

Proof. Since $-x-y=-a u, x-y=a v$, then $2 x=(x-y)-(-x-y)=a(u+v)$. Therefore $\|u+v\|=2\|x\| / a=2 / a$. Similarly, we can prove $\|u-v\|=2 / a$.

Proposition 2.5. $S\left(X_{2}\right)$ is affinely homeomorphic to a parallelogram if and only if $E\left(X_{2}\right)=$ 8 , or $f\left(X_{2}\right)=2$.

Proposition 2.6. If $S\left(X_{2}\right)$ is affinely homeomorphic to a normal hexagon, then $E\left(X_{2}\right) \geq 5$, and $f\left(X_{2}\right) \leq 32 / 9$.

Lemma 2.7 [7]. Let $x, y \in S(X)$, and $\|x+y\| / 2>1-\epsilon$, then for any $z \in[x, y]$, the line segment connecting $x$ and $y,\|z\|>1-2 \epsilon$.

Theorem 2.8. A Banach space with $E\left(X_{2}\right)<8$, or $f(X)>2$ is uniformly nonsquare.

Proof. Suppose $X$ is not uniformly nonsquare. For any $\epsilon>0$, there exist $x, y \in S(X)$ such that both $\|x+y\|$ and $\|x-y\|>2-\epsilon / 2$ [9]. Then, both $\|x-y\|^{2}$ and $\|x-y\|^{2} \geq 4-2 \epsilon$. Since $\epsilon$ can be arbitrarily small we have $E(X)=\sup \left\{\|x+y\|^{2}+\|x-y\|^{2}, x \in S(X), y \in\right.$ $S(X)\}=8$.

If $\|x+y\| \neq\|x-y\|$, take $z$ such that $y$ and $z$ are on the same arc $\kappa$ from $x$ to $-x$, and $\|x+z\|=\|x-z\|$. From Lemma 1.5 both $\|x+z\|$ and $\|x-z\|>2-\epsilon / 2$. Let $u$ and $v$ be normalization of $x+z$ and $x-z$ respectively. From Lemma 2.4, both $\|u+v\|$ and 
$\|u-v\| \leq 2 /(2-\epsilon / 2) \leq 1+\epsilon / 2$. Since $\epsilon$ can be arbitrarily small we have $f(X)=\inf \{\| x+$ $\left.y\left\|^{2}+\right\| x-y \|^{2}, x \in S(X), y \in S(X)\right\}=2$.

\section{Parameters $E(X)$ and $f(X)$, and normal structure}

Lemma 3.1. Let $x \in S(X)$ and $\|y\|>1$, then $\|x+t(y-x)\|$ is an increasing function of $t \in(1, \infty)$.

Proof. It is clear that $\|x+t(y-x)\|>1$ for any $t>1$. Otherwise

$$
\begin{aligned}
\|y\| & =\left\|\frac{x+t(y-x)}{t}+\frac{(t-1) x}{t}\right\| \\
& \leq \frac{\|x+t(y-x)\|}{t}+\frac{(t-1)\|x\|}{t} \\
& \leq \frac{1}{t}+\frac{t-1}{t}=1 .
\end{aligned}
$$

If there are $t_{1}>t_{2}>1$ such that $1 \leq a=\left\|x+t_{1}(y-x)\right\|<\left\|x+t_{2}(y-x)\right\|$, then

$$
\begin{aligned}
\left\|x+t_{2}(y-x)\right\| & =\left\|\frac{t_{2}\left[x+t_{1}(y-x)\right]}{t_{1}}+\frac{\left(t_{1}-t_{2}\right) x}{t_{1}}\right\| \\
& \leq \frac{t_{2}\left\|x+t_{1}(y-x)\right\|}{t_{1}}+\frac{\left(t_{1}-t_{2}\right)\|x\|}{t_{1}} \\
& =\frac{t_{2} a}{t_{1}}+\frac{t_{1}-t_{2}}{t_{1}} \leq \frac{t_{2} a}{t_{1}}+\frac{\left(t_{1}-t_{2}\right) a}{t_{1}}=a .
\end{aligned}
$$

This is a contradiction.

Theorem 3.2. A Banach space $X$ with $E(X)<5$, or $f(X)>32 / 9$ has normal structure.

Proof. Suppose $X$ does not have normal structure, then there exist $x_{1}, x_{2}$ and $x_{3} \in S(X)$ such that $x_{1}=x_{2}-x_{3},\left\|\left(x_{1}+x_{2}\right) / 2\right\|>1-\epsilon$, and $\left\|\left(x_{3}+\left(-x_{1}\right)\right) / 2\right\|>1-\epsilon$ by Lemma 1.3 . Then, $\left\|x_{3}-x_{1}\right\|^{2}>4-8 \epsilon$ and $\left\|x_{3}+x_{1}\right\|^{2}=\left\|x_{2}\right\|^{2}=1$. Since $\epsilon$ can be arbitrarily small we have $E(X)=\sup \left\{\|x+y\|^{2}+\|x-y\|^{2}, x \in S(X), y \in S(X)\right\} \geq 5$. The first assert is approved.

To prove the second assert, let $y=\left(x_{2}+x_{3}\right) / 2$, then $y-x_{1}=\left(x_{2}+x_{3}\right) / 2-x_{1}=\left(x_{2}+\right.$ $\left.x_{3}-2 x_{1}\right) / 2=\left(x_{1}+x_{3}+x_{3}-2 x_{1}\right) / 2=\left(2 x_{3}-x_{1}\right) / 2=3 / 2\left(2 x_{3} / 3+\left(-x_{1}\right) / 3\right)$. Since $\|\left(x_{3}+\right.$ $\left.\left(-x_{1}\right)\right) / 2 \|>1-\epsilon$ and $2 x_{3} / 3+\left(-x_{1}\right) / 3 \in\left[-x_{1}, x_{3}\right]$, by Lemma 2.7 we have $\left\|y-x_{1}\right\|=$ $3 / 2\left\|2 x_{3} / 3+\left(-x_{1}\right) / 3\right\|>3 / 2(1-2 \epsilon)$. Let $y^{\prime}$ be normalization of $y=\left(x_{2}+x_{3}\right) / 2$. From Lemma 3.1, $\left\|y^{\prime}-x_{1}\right\| \geq\left\|y-x_{1}\right\|>3 / 2(1-2 \epsilon)$.

Similarly we can prove $\left\|y^{\prime}+x_{1}\right\| \geq\left\|y+x_{1}\right\|>3 / 2(1-2 \epsilon)$.

If $\left\|y^{\prime}+x_{1}\right\| \neq\left\|y^{\prime}-x_{1}\right\|$, let $z \in S(X)$ such that $\left\|z+x_{1}\right\|=\left\|z-x_{1}\right\|$. From Lemma 1.5 we have $\left\|z+x_{1}\right\|=\left\|z-x_{1}\right\|>3 / 2(1-2 \epsilon)$. Let $u, v$ be the normalization of $z+x_{1}, z-$ $x_{1}$ respectively, by Lemma 2.4 we have both $\|u+v\|$ and $\|u-v\|<2 /(3 / 2(1-2 \epsilon))<$ $4 / 3(1+4 \epsilon)$. Since $\epsilon$ can be arbitrarily small, we have $f(X)=\inf \left\{\|x+y\|^{2}+\|x-y\|^{2}, x \in\right.$ $S(X), y \in S(X)\}=2(4 / 3)^{2}=32 / 9$. 


\section{Inequalities of parameters for some classical spaces}

Proposition 4.1. $E(H)=e(X)=F(X)=f(X)=4$, where $H$ is a Hilbert space.

This is a direct result of parallelogram law: $\|x+y\|^{2}+\|x-y\|^{2}=2\left(\|x\|^{2}+\|y\|^{2}\right)$ for all $x, y \in H$, in the Hilbert spaces.

Theorem 4.2. Let $p, q \geq 1$ and $1 / p+1 / q=1$, then

(i) $f\left(l_{p}\right)=2^{1+2 / q}, F\left(l_{p}\right) \leq 2^{1+2 / p} \leq e\left(l_{p}\right) \leq E\left(l_{p}\right), 1<p \leq 2$;

(ii) $f\left(l_{p}\right) \leq F\left(l_{p}\right) \leq 2^{1+2 / p} \leq e\left(l_{p}\right), E\left(l_{p}\right)=2^{1+2 / q}, p>2$.

Proof. (i) To prove $e\left(l_{p}\right) \geq 2^{1+2 / p}$, for any $0<\delta<1$ and $y=\left(y_{1}, y_{2}, y_{3}, \ldots, y_{n}, \ldots\right) \in S\left(l_{p}\right)$ take $N$ such that $\left|y_{N}\right|<\delta$. Let $e_{N}=(0,0, \ldots, 0,1,0, \ldots)$, where the $N$ th entry is 1 and others are 0 . Since $(1-x)^{p} \geq 1-p x$ for $0 \leq x \leq 1$ and $p>1$, we have

$$
\begin{aligned}
|| y \pm e_{N}||^{p}= & \left|y_{1}\right|^{p}+\left|y_{2}\right|^{p}+\cdots+\left|y_{N} \pm 1\right|^{p}+\left|y_{N+1}\right|^{p}+\cdots \\
\geq & \left|y_{1}\right|^{p}+\left|y_{2}\right|^{p}+\cdots+|1-| y_{N}||^{p}+\left|y_{N+1}\right|^{p}+\cdots \\
\geq & \left(1+\left|y_{1}\right|^{p}+\left|y_{2}\right|^{p}+\cdots+\left|y_{N}\right|^{p}+\left|y_{N+1}\right|^{p}+\cdots\right) \\
& -p\left|y_{N}\right|-\left|y_{N}\right|^{p} \geq 2-2 p \delta .
\end{aligned}
$$

Since $\delta$ can be arbitrarily small, by definition of $\alpha(y), \alpha(y) \geq 2 \cdot 2^{2 / p}=2^{1+2 / p}$. Since $y \in S\left(l_{p}\right)$ is arbitrary, by definition of $e(X)$, we have $e\left(l_{p}\right) \geq 2^{1+2 / p}$.

To prove $F\left(l_{p}\right) \leq 2^{1+2 / p}$, we need the inequality: $(1+x)^{p} \leq 1+2 p x$ if $x$ is small enough and $p>1$. For any $y=\left(y_{1}, y_{2}, y_{3}, \ldots, y_{n}, \ldots\right) \in S\left(l_{p}\right)$.

Take $N$ such that $\left|y_{N}\right|<\delta<1$. Let $e_{N}=(0,0, \ldots, 0,1,0, \ldots)$, where the $N$ th entry is 1 and others are 0 , we have

$$
\begin{aligned}
|| y \pm e_{N}||^{p}= & \left|y_{1}\right|^{p}+\left|y_{2}\right|^{p}+\cdots+\left|y_{N} \pm 1\right|^{p}+\left|y_{N+1}\right|^{p}+\cdots \\
\leq & \left|y_{1}\right|^{p}+\left|y_{2}\right|^{p}+\cdots+|1+| y_{N}||^{p}+\left|y_{N+1}\right|^{p}+\cdots \\
\leq & \left(\left|y_{1}\right|^{p}+\left|y_{2}\right|^{p}+\cdots+\left|y_{N}\right|^{p}+\left|y_{N+1}\right|^{p}+\cdots\right) \\
& +1+2 p\left|y_{N}\right| \leq 2+2 p \delta .
\end{aligned}
$$

Since $\delta$ can be arbitrarily small, by definition of $\beta(y), \beta(y) \leq 2 \cdot 2^{2 / p}=2^{1+2 / p}$. Since $y \in S\left(l_{p}\right)$ is arbitrary, by definition of $F(X)$, we have $F\left(l_{p}\right) \leq 2^{1+2 / p}$.

To prove $f\left(l_{p}\right)=2^{1+2 / q}$, let $x=2^{-1 / p}(1,1,0, \ldots, 0, \ldots), y=2^{-1 / p}(1,-1,0, \ldots, 0, \ldots) \in$ $S\left(l_{p}\right)$. Then $\|x+y\|^{2}+\|x-y\|^{2}=2^{-2 / p}\left(\|(2,0,0, \ldots, 0, \ldots)\|_{l_{p}}^{2}+\|(0,2,0, \ldots, 0, \ldots)\|_{l_{p}}^{2}\right)=2$. $2^{-2 / p} \cdot 4=2^{3-2 / p}=2^{1+2 / q}$. From definition of $f(X)$, we have $f\left(l_{p}\right) \leq 2^{1+2 / q}$.

On the other hand, by using Lagrange multipliers in basic calculus, the function $u^{2}+$ $v^{2}$, under the constraint $u^{p}+v^{p}=a$ for $1<p \leq 2$, assumes its minimum $2^{1-2 / p} \cdot a^{2 / p}$ at $u=v=(a / 2)^{1 / p}$.

The Clarkson inequality $[3,4]:\|x+y\|^{p}+\|x-y\|^{p} \geq(\|x\|+\|y\|)^{p}+\|\| x\|-\| y \|\left.\right|^{p}$, for all $x, y \in X$, implies that $\|x+y\|^{p}+\|x-y\|^{p} \geq 2^{p}$, for all $x, y \in S\left(l_{p}\right)$. Therefore $\| x+$ $y\left\|^{2}+\right\| x-y \|^{2} \geq 2^{1-2 / p} \cdot\left(2^{p}\right)^{2 / p}=2^{3-2 / p}=2^{1+2 / q}$, for all $x, y \in S\left(l_{p}\right)$. By definition of $f(X)$ we have $f\left(l_{p}\right) \geq 2^{1+2 / q}$.

Finally, $f\left(l_{p}\right)=2^{1+2 / q}$. 
(ii) To prove $F\left(l_{p}\right) \leq 2^{1+2 / p}$, we need the inequality: $(1+x)^{p} \leq 1+2 p x$ if $x$ is small enough and $p>1$. For any $y=\left(y_{1}, y_{2}, y_{3}, \ldots, y_{n}, \ldots\right) \in S\left(l_{p}\right)$.

Take $N$ such that $\left|y_{N}\right|<\delta<1$. Let $e_{N}=(0,0, \ldots, 0,1,0, \ldots)$, where the $N$ th entry is 1 and others are 0 , we have

$$
\begin{aligned}
\| y \pm\left. e_{N}\right|^{p}= & \left|y_{1}\right|^{p}+\left|y_{2}\right|^{p}+\cdots+\left|y_{N} \pm 1\right|^{p}+\left|y_{N+1}\right|^{p}+\cdots \\
\leq & \left|y_{1}\right|^{p}+\left|y_{2}\right|^{p}+\cdots+|1+| y_{N}||^{p}+\left|y_{N+1}\right|^{p}+\cdots \\
\leq & \left(\left|y_{1}\right|^{p}+\left|y_{2}\right|^{p}+\cdots+\left|y_{N}\right|^{p}+\left|y_{N+1}\right|^{p}+\cdots\right) \\
& +1+2 p\left|y_{N}\right| \leq 2+2 p \delta .
\end{aligned}
$$

Since $\delta$ can be arbitrarily small, by definition of $\beta(y), \beta(y) \leq 2 \cdot 2^{2 / p}=2^{1+2 / p}$. Since $y \in S\left(l_{p}\right)$ is arbitrary, by definition of $F(X)$, we have $F\left(l_{p}\right) \leq 2^{1+2 / p}$.

To prove $e\left(l_{p}\right) \geq 2^{1+2 / p}$, for any $0<\delta<1$ and $y=\left(y_{1}, y_{2}, y_{3}, \ldots, y_{n}, \ldots\right) \in S\left(l_{p}\right)$ take $N$ such that $\left|y_{N}\right|<\delta$. Let $e_{N}=(0,0, \ldots, 0,1,0, \ldots)$, where the $N$ th entry is 1 and others are 0 . Since $(1-x)^{p} \geq 1-p x$ for $0 \leq x \leq 1$ and $p>1$, we have

$$
\begin{aligned}
\| y \pm\left. e_{N}\right|^{p}= & \left|y_{1}\right|^{p}+\left|y_{2}\right|^{p}+\cdots+\left|y_{N} \pm 1\right|^{p}+\left|y_{N+1}\right|^{p}+\cdots \\
\geq & \left|y_{1}\right|^{p}+\left|y_{2}\right|^{p}+\cdots+|1-| y_{N}||^{p}+\left|y_{N+1}\right|^{p}+\cdots \\
\geq & \left(1+\left|y_{1}\right|^{p}+\left|y_{2}\right|^{p}+\cdots+\left|y_{N}\right|^{p}+\left|y_{N+1}\right|^{p}+\cdots\right) \\
& -p\left|y_{N}\right|-\left|y_{N}\right|^{p} \geq 2-2 p \delta .
\end{aligned}
$$

Since $\delta$ can be arbitrarily small, by definition of $\alpha(y), \alpha(y) \geq 2 \cdot 2^{2 / p}=2^{1+2 / p}$. Since $y \in S\left(l_{p}\right)$ is arbitrary, by definition of $e(X)$, we have $e\left(l_{p}\right) \geq 2^{1+2 / p}$.

To prove $E\left(l_{p}\right)=2^{1+2 / q}$, let $x=2^{-1 / p}(1,1,0, \ldots, 0, \ldots), y=2^{-1 / p}(1,-1,0, \ldots, 0, \ldots)$ $\in S\left(l_{p}\right)$. Then $\|x+y\|^{2}+\|x-y\|^{2}=2^{-2 / p}\left(\|(2,0,0, \ldots, 0, \ldots)\|_{l_{p}}^{2}+\|\left(0,2,0, \ldots, 0, \ldots \|_{l_{p}}\right)^{2}\right)=$ $2 \cdot 2^{-2 / p} \cdot 4=2^{3-2 / p}=2^{1+2 / q}$. From definition of $E(X)$, we have $E\left(l_{p}\right) \geq 2^{1+2 / q}$.

On the other hand, by using Lagrange multipliers in basic calculus, the function $u^{2}+$ $v^{2}$, under the constraint $u^{p}+v^{p}=a$ for $p>2$, assumes its maximum $2^{1-2 / p} \cdot a^{2 / p}$ at $u=$ $v=(a / 2)^{1 / p}$.

The Clarkson inequality: $\|x+y\|^{p}+\|x-y\|^{p} \leq(\|x\|+\|y\|)^{p}+|\|x\|-\|y\||^{p}$, for all $x, y \in X$, implies that $\|x+y\|^{p}+\|x-y\|^{p} \leq 2^{p}$, for all $x, y \in S\left(l_{p}\right)$. Therefore $\|x+y\|^{2}+$ $\|x-y\|^{2} \leq 2^{1-2 / p} \cdot\left(2^{p}\right)^{2 / p}=2^{3-2 / p}=2^{1+2 / q}$, for all $x, y \in S\left(l_{p}\right)$. By the definition of $E(X)$ we have $E\left(l_{p}\right) \leq 2^{1+2 / q}$.

Finally, we have $E\left(l_{p}\right)=2^{1+2 / q}$.

Theorem 4.3. Let $p, q \geq 1$ and $1 / p+1 / q=1$, then

(i) $f\left(L_{p}[0,1]\right)=F\left(L_{p}[0,1]\right)=2^{1+2 / q}$, $2^{1+2 / p} \leq e\left(L_{p}[0,1]\right) \leq E\left(L_{p}[0,1]\right), 1<p \leq 2 ;$

(ii) $f\left(L_{p}[0,1]\right) \leq F\left(L_{p}[0,1]\right) \leq 2^{1+2 / p}$, $e\left(L_{p}[0,1]\right)=E\left(L_{p}[0,1]\right)=2^{1+2 / q}, p>2$.

Proof. (i) To prove $e\left(L_{p}[0,1]\right) \geq 2^{1+2 / p}$, let $x(t) \in S\left(L_{p}[0,1]\right)$.

For any $0<\delta<1$, we can find a $\gamma>0$, a subset $E \subseteq[0,1]$ with $m E=\gamma$ and $\int_{E}|x(t)|^{p} d t<$ $\delta$, such that $|x(t)|<\gamma^{-1 / p}(t \in E)$. To prove the existence of such a set $E$, for the given 
$\gamma>0$ it is easy to take $E \subseteq[0,1]$ such that $m E=\gamma$ and $\int_{E}|x(t)|^{p} d t<\delta$. Consider $E_{1}=$ $\left\{t \in E,|x(t)|<\gamma^{-1 / p}\right\}$. We claim then $\gamma \geq \gamma_{1}=m E_{1}>0$. Otherwise, if $m E_{1}=0$ then $\int_{E}|x(t)|^{p} d t \geq 1$, and hence $\delta \geq 1$, this is a contradiction with $0<\delta<1$. We now can take $E_{1}$ as $E, \gamma_{1}$ as $\gamma$, and for $t \in E_{1},|x(t)|<\gamma^{-1 / p}$ implies $|x(t)|<\gamma_{1}^{-1 / p}$. Let

$$
z(t)= \begin{cases}\gamma^{-1 / p}, & t \in E \\ 0, & \text { otherwise }\end{cases}
$$

By using the inequality: $(1-x)^{p} \geq 1-p x$ for $0 \leq x \leq 1, p>1$, and Hölder's inequality:

$$
\begin{aligned}
\int_{E}|x(t)| d t \leq & \left(\int_{E} 1^{q} d t\right)^{1 / q}\left(\int_{E}|x(t)|^{p} d t\right)^{1 / p}, \\
|| x(t) \pm\left. z(t)\right|^{p}= & \int_{E}\left|x(t) \pm \gamma^{-1 / p}\right|^{p} d t+\int_{[0,1]-E}|x(t)|^{p} d t \\
\geq & \gamma^{-1} \int_{E}\left|1 \pm \gamma^{1 / p} x(t)\right|^{p} d t+1-\delta \geq \gamma^{-1} \int_{E}\left(1-\gamma^{1 / p}|x(t)|\right)^{p} d t \\
& +1-\delta \geq \gamma^{-1} \int_{E}\left(1-p \gamma^{1 / p}|x(t)|\right) d t+1-\delta \\
= & \gamma^{-1} \gamma-p \gamma^{-1+1 / p} \int_{E}|x(t)| d t+1-\delta \\
\geq & 1+1-p \gamma^{-1+1 / p+1 / q}\left(\int_{E}|x(t)|^{p} d t\right)^{1 / p}-\delta \geq 2-p \delta^{1 / p}-\delta .
\end{aligned}
$$

Since $\delta$ is arbitrary, $\alpha(x(t)) \geq 2 \cdot 2^{2 / p}=2^{1+2 / p}$. Since $x(t)$ is also arbitrary, we have $e\left(L_{p}[0,1]\right) \geq 2^{1+2 / p}$.

To prove $F\left(L_{p}[0,1]\right) \leq 2^{1+2 / q}$, let $x(t) \in S\left(L_{p}[0,1]\right)$, then $\int_{0}^{1}|x(t)|^{p} d t=1$. Take $\gamma \in$ $[0,1]$, such that $\int_{0}^{\gamma}|x(t)|^{p} d t=1 / 2$, then $\int_{\gamma}^{1}|x(t)|^{p} d t=1 / 2$ also. Let

$$
y(t)= \begin{cases}x(t), & 0 \leq t<\gamma \\ -x(t), & \gamma \leq t \leq 1\end{cases}
$$

then $y(t) \in S\left(L_{p}[0,1]\right)$, and $\|x(t)+y(t)\|^{p}=\int_{0}^{\gamma} 2^{p}|x(t)|^{p} d t=2^{p-1}$.

Similarly $\|x(t)-y(t)\|^{p}=2^{p-1}$ too.

By definition of $\beta(x(t)), \beta(x(t)) \leq 2 \cdot 2^{(2 p-2) / p}=2^{3-2 / p}=2^{1+2 / q}$. Since $x(t)$ is arbitrary, we have $F\left(L_{p}[0,1]\right) \leq 2^{1+2 / q}$.

On the other hand, recall the function $u^{2}+v^{2}$, under the constraint $u^{p}+v^{p}=a$ for $1<p \leq 2$, assumes its minimum $2^{1-2 / p} \cdot a^{2 / p}$ at $u=v=(a / 2)^{1 / p}$.

The Clarkson inequality [3, 4]: $\|x+y\|^{p}+\|x-y\|^{p} \geq(\|x\|+\|y\|)^{p}+\|\| x\|-\| y \|\left.\right|^{p}$, for all $x, y \in X$, implies that $\|x+y\|^{p}+\|x-y\|^{p} \geq 2^{p}$, for all $x, y \in S\left(l_{p}\right)$. Therefore $\| x+$ $y\left\|^{2}+\right\| x-y \|^{2} \geq 2^{1-2 / p} \cdot\left(2^{p}\right)^{2 / p}=2^{3-2 / p}=2^{1+2 / q}$, for all $x, y \in S\left(L_{p}[0,1]\right)$. By definition of $f(X)$, we have $f\left(L_{p}[0,1]\right) \geq 2^{1+2 / q}$.

From $2^{1+2 / q} \leq f\left(L_{p}[0,1]\right) \leq F\left(L_{p}[0,1]\right) \leq 2^{1+2 / q}$, we have $f\left(L_{p}[0,1]\right)=F\left(L_{p}[0,1]\right)=$ $2^{1+2 / q}$. 
(ii) To prove $F\left(L_{p}[0,1]\right) \leq 2^{1+2 / p}$, for all $y(t) \in S\left(L_{p}[0,1]\right)$, take $\delta>0$ such that $(1+$ $\delta)^{p}<1+2 p \delta$. We can find $\gamma>0$ with $\int_{0}^{\gamma}|x(t)|^{p} d t<\delta$.

Let

$$
y(t)= \begin{cases}\gamma^{-1 / p}, & 0 \leq t<\gamma \\ 0, & \gamma \leq t \leq 1\end{cases}
$$

then by Minkowski inequality:

$$
\begin{aligned}
\left(\int_{a}^{b}|x(t)+y(t)|^{p} d t\right)^{1 / p} & \leq\left(\int_{a}^{b}|x(t)|^{p} d t\right)^{1 / p}+\left(\int_{a}^{b}|y(t)|^{p} d t\right)^{1 / p} \\
\|x(t) \pm y(t)\|^{p} & =\int_{0}^{\gamma}\left|x(t) \pm \gamma^{-1 / p}\right|^{p} d t+\int_{\gamma}^{1}|x(t)|^{p} d t \\
& \leq\left[\left(\int_{0}^{\gamma}|x(t)|^{p} d t\right)^{1 / p}+\left(\int_{0}^{\gamma}\left|\gamma^{-1 / p}\right|^{p} d t\right)^{1 / p}\right]^{p}+1 \\
& \leq\left(\delta^{1 / p}+1\right)^{p}+1 .
\end{aligned}
$$

Since $\delta$ is arbitrary, from definition of $\beta(x(t)), \beta(x(t)) \leq 2 \cdot 2^{2 / p}=2^{1+2 / p}$. Since $x(t)$ is also arbitrary, we have $F\left(L_{p}[0,1]\right) \leq 2^{1+2 / p}$.

To prove $e\left(L_{p}[0,1]\right) \geq 2^{1+2 / q}$, let $x(t) \in S\left(L_{p}[0,1]\right)$, then $\int_{0}^{1}|x(t)|^{p} d t=1$. Take $\gamma \in$ $[0,1]$, such that $\int_{0}^{\gamma}|x(t)|^{p} d t=1 / 2$, then $\int_{\gamma}^{1}|x(t)|^{p} d t=1 / 2$ also. Let

$$
y(t)= \begin{cases}x(t), & 0 \leq t<\gamma \\ -x(t), & \gamma \leq t \leq 1\end{cases}
$$

then $y(t) \in S\left(L_{p}[0,1]\right)$, and $\|x(t)+y(t)\|^{p}=\int_{0}^{\gamma} 2^{p}|x(t)|^{p} d t=2^{p-1}$.

Similarly $\|x(t)-y(t)\|^{p}=2^{p-1}$ too.

By definition of $\alpha(x(t)), \alpha(x(t)) \geq 2 \cdot 2^{(2 p-2) / p}=2^{3-2 / p}=2^{1+2 / q}$. Since $x(t)$ is arbitrary we have $e\left(L_{p}[0,1]\right) \geq 2^{1+2 / q}$.

On the other hand, recall the function $u^{2}+v^{2}$, under the constraint $u^{p}+v^{p}=a$ for $p>2$, assumes its maximum $2^{1-2 / p} \cdot a^{2 / p}$ at $u=v=(a / 2)^{1 / p}$.

The Clarkson inequality for $p \geq 2$ : $\|x+y\|^{p}+\|x-y\|^{p} \leq(\|x\|+\|y\|)^{p}+\|\| x \|$ - $\|y\| \|^{p}$, for all $x, y \in X$, implies that $\|x+y\|^{p}+\|x-y\|^{p} \leq 2^{p}$, for all $x, y \in S\left(l_{p}\right)$. Therefore $\|x+y\|^{2}+\|x-y\|^{2} \leq 2^{1-2 / p} \cdot\left(2^{p}\right)^{2 / p}=2^{3-2 / p}=2^{1+2 / q}$ for all $x, y \in S\left(L_{p}[0,1]\right)$. By the definition of $E(X)$ we have $E\left(L_{p}[0,1]\right) \leq 2^{1+2 / q}$.

From $2^{1+2 / q} \leq e\left(L_{p}[0,1]\right) \leq E\left(L_{p}[0,1]\right) \leq 2^{1+2 / q}$, we have $e\left(L_{p}[0,1]\right)=E\left(L_{p}[0,1]\right)=$ $2^{1+2 / q}$.

\section{The parameters and uniform normal structure}

Let $U$ be an ultrafilter on an index set $I$. Let $\left\{X_{i}\right\}_{i \in I}$ be a family of Banach spaces and let $l_{\infty}\left(I, X_{i}\right)$ denote the subspace of the product space equipped with the norm $\left\|\left(x_{i}\right)\right\|=$ $\sup _{i \in I}\left\|x_{i}\right\|<\infty$. 
Definition 5.1 [13]. Let $U$ be an ultrafilter on $I$ and let $N_{U}=\left\{\left(x_{i}\right) \in l_{\infty}\left(I, X_{i}\right): \lim _{U}\left\|x_{i}\right\|\right.$ $=0\}$. The ultraproduct of $\left\{X_{i}\right\}_{i \in I}$ is the quotient space $l_{\infty}\left(I, X_{i}\right) / N_{U}$ equipped with the quotient norm.

We will use $\left(x_{i}\right)_{U}$ to denote the element of the ultraproduct. It follows from (ii) of the fact, and the definition of quotient norm that

$$
\left\|\left(x_{i}\right)_{U}\right\|=\lim _{U}\left\|x_{i}\right\|
$$

In the following we will restrict our index set $I$ to be $N$, the set of natural numbers, and let $X_{i}=X, i \in N$ for some Banach space $X$. For an ultrafilter $U$ on $N$, we use $X_{U}$ to denote the ultraproduct.

Theorem 5.2. For any Banach space $X$, and for any nontrivial ultrafilter $U$ on $N, K\left(X_{U}\right)=$ $K(X)$, where $K$ stands for either $f, F$, e, or $E$.

Proof. We first prove this theorem for $K=f$, the proof for $K=E$ is similar.

For any $\delta>0$, from definition of $f(X)$, there exists a two-dimensional subspace $X_{2} \subseteq$ $X$, and $x, y \in S\left(X_{2}\right)$ such that $\|x+y\|^{2}+\|x-y\|^{2}<f(X)+\delta$.

Let $x_{i}=x$, and $y_{i}=y$, for all $i \in N$. Then $\left(x_{i}\right)_{U},\left(y_{i}\right)_{U} \in S\left(\left(X_{U}\right)_{2}\right)$, where $\left(X_{U}\right)_{2}$ is a two-dimensional subspace, spanned by $\left(x_{i}\right)_{U}$ and $\left(y_{i}\right)_{U}$, of $X_{U}$. Then $\left\|\left(x_{i}\right)_{U}+\left(y_{i}\right)_{U}\right\|^{2}+$ $\left\|\left(x_{i}\right)_{U}-\left(y_{i}\right)_{U}\right\|^{2}=\|x+y\|^{2}+\|x-y\|^{2}<f(X)+\delta$. Therefore $f\left(X_{U}\right)<f(X)+\delta$.

Since $\delta$ can be arbitrarily small, we proved $f\left(X_{U}\right) \leq f(X)$.

To prove the reverse inequality, we choose a $\left(X_{U}\right)_{2} \subseteq X_{U},\left(x_{i}\right)_{U},\left(y_{i}\right)_{U} \in S\left(\left(X_{U}\right)_{2}\right)$ such that $\left\|\left(x_{i}\right)_{U}+\left(y_{i}\right)_{U}\right\|^{2}+\left\|\left(x_{i}\right)_{U}-\left(y_{i}\right)_{U}\right\|^{2}<f\left(X_{U}\right)+\delta$.

From (5.1) above, the sets

$I=\left\{i \in N:||\left|x_{i}\right||-1|<\delta\right\}$,

$J=\left\{i \in N:||\left|y_{i} \|-1\right|<\delta\right\}$,

$L=\left\{i \in N:\left\|x_{i}+y_{i}\right\|^{2}+\left\|x_{i}-y_{i}\right\|^{2}<f\left(X_{U}\right)+\delta\right\}$ are all in $U$. So the intersection $I \cap J \cap L$ is in $U$ too, and is hence not empty.

Let $i \in I \cap J \cap L$, we have $\left|\left\|x_{i}\right\|-1\right|<\delta$, $\left|\left\|y_{i}\right\|-1\right|<\delta$, and $\left\|x_{i}+y_{i}\right\|^{2}+\left\|x_{i}-y_{i}\right\|^{2}<$ $f\left(X_{U}\right)+\delta$. Let $x_{i}^{\prime}, y_{i}^{\prime} \in S\left(X_{2}\right)$ be the normalization of $x_{i}, y_{i}$ respectively. Since $\left\|x_{i}^{\prime}-x_{i}\right\|$ and $\left\|y_{i}^{\prime}-y_{i}\right\| \leq \delta$, then $\left\|x_{i}^{\prime}+y_{i}^{\prime}\right\| \leq\left\|x_{i}+y_{i}\right\|+2 \delta,\left\|x_{i}^{\prime}-y_{i}^{\prime}\right\| \leq\left\|x_{i}-y_{i}\right\|+2 \delta$, and therefore $\left\|x_{i}^{\prime}+y_{i}^{\prime}\right\|^{2}+\left\|x_{i}^{\prime}-y_{i}^{\prime}\right\|^{2} \leq\left\|x_{i}+y_{i}\right\|^{2}+\left\|x_{i}-y_{i}\right\|^{2}+18 \delta<f\left(X_{U}\right)+19 \delta$. Hence $f(X)<$ $f\left(X_{U}\right)+19 \delta$. Since $\delta$ can be arbitrarily small, $f(X) \leq f\left(X_{U}\right)$.

We then prove this theorem for $K=F$, the proof for $K=e$ is similar.

For any $\delta>0$, from definition of $F(X)$, there exists a $x \in S(X)$ such that $\|x+y\|^{2}+$ $\|x-y\|^{2}>F(X)-\delta$ for all $y \in S(X)$.

Let $x_{i}=x$, for all $i \in N$, then $\left(x_{i}\right)_{U} \in S\left(\left(X_{U}\right)\right)$. For any $\left(y_{i}\right)_{U} \in X_{U}$, from definition of the norm in $X_{U}$, we may assume that $y_{i} \in S(X)$, for all $i \in N$. Therefore

$$
\begin{aligned}
& \left\|\left(x_{i}\right)_{U}+\left(y_{i}\right)_{U}\right\|^{2}+\left\|\left(x_{i}\right)_{U}-\left(y_{i}\right)_{U}\right\|^{2} \\
& \quad=\left\|\left(x+y_{i}\right)_{U}\right\|^{2}+\left\|\left(x-y_{i}\right)_{U}\right\|^{2} \\
& \quad=\left(\left\|x+y_{i}\right\|^{2}+\left\|x-y_{i}\right\|^{2}\right)_{U}>F(X)-\delta .
\end{aligned}
$$

Therefore $F\left(X_{U}\right)>F(X)-\delta$. 
Since $\delta$ can be arbitrarily small, we proved $F\left(X_{U}\right) \geq F(X)$.

To prove the reverse inequality, for any $\delta>0$, from definition of $F\left(X_{U}\right)$ there exists a $\left(x_{i}\right)_{U} \in S\left(\left(X_{U}\right)\right)$ such that $\left\|\left(x_{i}\right)_{U}+\left(y_{m}\right)_{U}\right\|^{2}+\left\|\left(x_{i}\right)_{U}-\left(y_{m}\right)_{U}\right\|^{2}>f\left(X_{U}\right)-\delta$ for all $\left(y_{m}\right)_{U} \in S\left(\left(X_{U}\right)\right)$. From definition of the norm in $X_{U}$, we may assume $x_{i}, y_{m} \in S(X)$, for all $i, m \in N$.

Let $L=\left\{i \in N:\left\|x_{i}+y_{m}\right\|^{2}+\left\|x_{i}-y_{m}\right\|^{2}>f\left(X_{U}\right)-\delta\right\}$, then $L$ is in $U$, and is hence not empty. Let $j \in L$, from (5.1) above, we have $\left\|x_{j}+y_{m}\right\|^{2}+\left\|x_{j}-y_{m}\right\|^{2}>f\left(X_{U}\right)-\delta$ for $y_{m} \in S(X)$. But $y_{m} \in S(X)$ may be any vector on $S(X)$. We have $\left\|x_{j}+y\right\|^{2}+\left\|x_{j}-y\right\|^{2}>$ $f\left(X_{U}\right)-\delta$ for all $y \in S(X)$. Therefore $f(X)>f\left(X_{U}\right)-\delta$. Since $\delta$ can be arbitrarily small, $f(X) \geq f\left(X_{U}\right)$.

Finally $f(X)=f\left(X_{U}\right)$.

Theorem 5.3. A Banach space $X$ with either $f(X)>32 / 9$ or $E(X)<5$ has uniform normal structure.

Proof. The idea of the proof is same as the proof of [7, Theorem 4.4].

\section{Acknowledgment}

The author would like to thank the referee and Professor B. Webber of Community College of Philadelphia for making the paper more readable.

\section{References}

[1] M. S. Brodskiĭ and D. P. Mil'man, On the center of a convex set, Doklady Akademii Nauk. SSSR (N.S.) 59 (1948), 837-840 (Russian).

[2] H. Busemann, The Geometry of Geodes, Academic Press, New York, 1955.

[3] J. A. Clarkson, Uniformly convex spaces, Transactions of the American Mathematical Society 40 (1936), no. 3, 396-414.

[4] M. M. Day, Normed Linear Spaces, 3rd ed., Ergebnisse der Mathematik und ihrer Grenzgebiete, vol. 21, Springer, New York, 1973.

[5] J. Gao, Normal hexagon and more general Banach spaces with uniform normal structure, Journal of Mathematics. Shuxue Zazhi 20 (2000), no. 3, 241-248.

[6] __ Normal structure, fixed points and related parameters in Banach spaces, Journal of Dynamical Systems and Geometric Theories 1 (2002), no. 1, 1-18.

[7] J. Gao and K.-S. Lau, On two classes of Banach spaces with uniform normal structure, Polska Akademia Nauk. Instytut Matematyczny. Studia Mathematica 99 (1991), no. 1, 41-56.

[8] J. García-Falset, Stability and fixed points for nonexpansive mappings, Houston Journal of Mathematics 20 (1994), no. 3, 495-506.

[9] R. C. James, Uniformly non-square Banach spaces, Annals of Mathematics. Second Series (2) 80 (1964), 542-550.

[10] W. A. Kirk, A fixed point theorem for mappings which do not increase distances, The American Mathematical Monthly 72 (1965), 1004-1006.

[11] E. M. Mazcuñán-Navarro, On the modulus of u-convexity of Ji Gao, Abstract and Applied Analysis 2003 (2003), no. 1, 49-54.

[12] J. J. Schäffer, Geometry of Spheres in Normed Spaces, Lecture Notes in Pure and Applied Mathematics, no. 20, Marcel Dekker, New York, 1976. 
Ji Gao 11

[13] B. Sims, "Ultra"-Techniques in Banach Space Theory, Queen's Papers in Pure and Applied Mathematics, vol. 60, Queen's University, Ontario, 1982.

[14] _ A class of spaces with weak normal structure, Bulletin of the Australian Mathematical Society 49 (1994), no. 3, 523-528.

Ji Gao: Department of Mathematics, Community College of Philadelphia, Philadelphia, PA 19130-3991, USA

E-mail address: igao@ccp.edu 